\title{
Angioscopic Evaluation of Neointimal Coverage of Coronary Stents
}

\author{
Yasumi Uchida • Yasuto Uchida
}

Published online: 4 August 2010

(C) The Author(s) 2010. This article is published with open access at Springerlink.com

\begin{abstract}
Drug-eluting stents (DES) reduce coronary restenosis significantly; however, late stent thrombosis (LST) occurs, which requires long-term antiplatelet therapy. Angioscopic grading of neointimal coverage of coronary stent struts was established, and it was revealed that neointimal formation is incomplete and prevalence of LST is higher in DES when compared to bare-metal stents. It was also observed that the neointima is thicker and LST is less frequent in paclitaxeleluting and zotarolimus-eluting stents than in sirolimuseluting stents. Many new stents were devised and they are now under experimental or clinical investigations to overcome the shortcomings of the stents that have been employed clinically. Endothelial cells are highly anti-thrombotic. Neoendothelial cell damage is considered to be caused by friction between the cells and stent struts due to the thin neointima between them which might act as a cushion. Therefore, development of a DES that causes an appropriate thickness (around $100 \mu \mathrm{m}$ ) of the neointima is a potential option with which to prevent neo-endothelial cell damage and consequent LST while preventing restenosis.
\end{abstract}

Keywords Bare-metal stent · Drug-eluting stent .

Neointimal coverage $\cdot$ Late stent thrombosis .

Neo-endothelial cell damage $\cdot$ Angioscopy

\footnotetext{
Y. Uchida $(\triangle)$

Japan Foundation for Cardiovascular Research,

2-30-17, Narashinodai,

Funabashi 274-0063, Japan

e-mail: uchiy@ta2.so-net.ne.jp

Y. Uchida

Cardiology, Toho University Ohmori Hospital,

Tokyo, Japan
}

\section{Introduction}

Restenosis after stent implantation is an overreaction of the wound healing response after vascular injury, characterized by inflammation, granulation, extra-matrix remodeling, progenitor cell migration and differentiation, and smooth muscle cell proliferation and migration and their consequent change into connective tissues.

Neointima formation on stent struts is essential for the vascular wound healing process [1]. Drug-eluting stents (DES) inhibit the mobilization and differentiation of progenitor cells of endothelial cells and smooth muscle cells, and thus not only inhibit restenosis but also impair neointima formation, which may lead to stent thrombosis.

DES have reduced restenosis significantly, but their shortcomings became evident, namely insufficient stent strut coverage by neointima, and consequent late-stent thrombosis (LST) or very LST, and consequent acute coronary syndrome that can occur on termination of antithrombotic therapy. This is a typical example of "a new therapeutic modality that has a new complication."

To improve the long-term prognosis in the DES era, development of new DES that accelerate normally functioning neo-endothelial cell formation and cause adequate smooth muscle and collagen fiber proliferation is required.

\section{Grading of Neointimal Coverage}

The thickness of the neointima on the stent struts can be measured in $\mu \mathrm{m}$ by optical coherence tomography (OCT), and it can be assessed by angioscopy based on whether or not the stent struts can be seen through the neointima. Therefore, the assessment of neointimal coverage by angioscopy is influenced by the composition of the neointima. Nevertheless, 
angioscopy is a powerful imaging modality to assess whether the stent struts are naked (uncovered) or not, the characteristics of the neointima, and whether a thrombus is formed on them. This imaging modality can clarify the compositions of a thrombus: namely red blood cells, platelets and fibrin, and fresh or old thrombus [2].

Initially, the grade of stent strut coverage by neointima was angioscopically classified into naked (reflects light, such as immediately after implantation), seen-through (covered by neointima but stent struts are visible), and not seen-through (covered by neointima and stent struts are not visible).

Later, Higo et al. [3•] and Oyabu et al. [4] classified neointimal coverage into grade 0 (not covered); grade 1 (covered by a thin layer); and grade 2 (buried under neointima). Kotani et al. [5] classified neointimal coverage into four grades: grade 0 (stent struts were exposed); grade 1 (struts bulged into the lumen, although they were covered by neointima); grade 2 (struts were imbedded but were seen translucently); and grade 3 (struts were fully embedded and not visible) (Fig. 1, Table 1). It is still not settled which of these grading systems is more adequate for evaluation of neointima.

\section{Comparison of Angioscopy and OCT in Evaluating Neointimal Coverage}

By comparing angioscopy and OCT images in patients, Tsujimoto [6] observed that stent struts were visible by
Table 1 Angioscopic grading of neointimal coverage

\begin{tabular}{lc}
\hline Grade 0 & Stent struts were exposed \\
Grade 1 & Struts bulged into the lumen, although \\
& they were covered by neointima \\
Grade 2 & Struts were embedded but were seen \\
translucently \\
Grade 3 \\
Struts were fully embedded and not \\
visible
\end{tabular}

Cited from Kotani et al. [5]

angioscopy when the neointimal thickness was within $130 \mu \mathrm{m}$ by OCT. This study connected angioscopic neointima grading and exact neointima thickness.

\section{Difference in Neointimal Coverage Between Bare-Metal Stents and DES}

Although restenosis rates have been markedly reduced by DES, it became evident that stent strut coverage by neointima was incomplete or much delayed in cases of DES when compared with bare-metal stents (BMS); stent struts were covered by neointima in $93 \%$ to $100 \%$ of BMS at 6 months $[7,8]$; grade 3 neointimal coverage was seen in $100 \%$ of BMS but in only $46 \%$ of sirolimuseluting stents (SES) at 6 months; and incomplete neointimal coverage was observed even at 2 years after SES implantation [9].
Fig. 1 Angioscopic grading of neointimal coverage of stent struts, and differences in incidence of each grade and late stent thrombosis between zotarolimus-eluting stents (ZES) and sirolimus-eluting stents (SES). (Adapted from Kotani et al. [5]; with permission.)

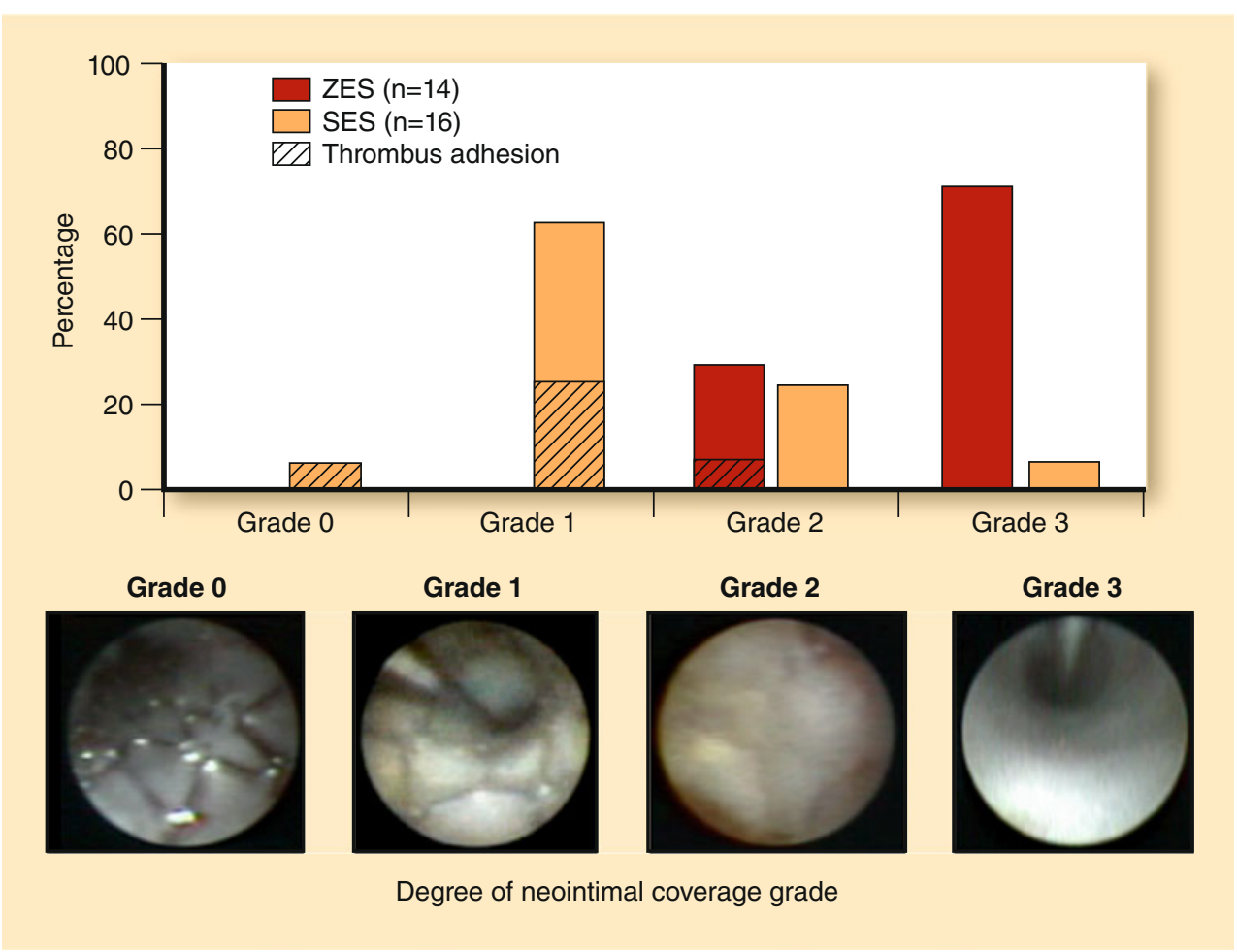




\section{Differences in Neointimal Coverage Among DES}

Awata et al. [10•*] observed that $71 \%$ of zotarolimus-eluting stents (ZES) showed grade 3 neointimal coverage, whereas $6 \%$ of SES showed grade 3 coverage at 8 months after deployment. They also observed grades 0 and 1 in SES but not in ZES [10••]. Similarly, the thickness of neointima in paclitaxel-eluting stents (PES) was much greater than in SES $[11,12]$. Incomplete neointimal coverage of SES has also been observed in $11 \%$ at 18 months [13].

Since the resolution was reported to be superior to intravascular ultrasound (IVUS) [14], OCT was used to evaluate neointimal coverage in DES. Compared with SES, PES has been found to have a larger neointimal thickness with fewer uncovered struts [15].

The findings of all of the studies mentioned above indicate neointimal formation is incomplete or delayed in SES compared with other DES. However, complete neointimal coverage on SES can be attained by IVUS-guided optimal sizing of SES [16].

Chieffo et al. [17] obtained neointima by directional coronary atherectomy from patients, and they found that smooth muscle phenotype was different among SES, PES, ZES, and TES (tacrolimus-eluting stent). Wilson et al. [18] observed in a swine model that persistent para-strut fibrin deposition was dominant in PES, whereas fibrin deposition within neointima and medial smooth muscle cell death were dominant in SES. These histological differences may participate in different neointima coverage among the DES.

\section{Heterogenicity of Neointimal Coverage in DES}

Kim et al. [19] observed by OCT that neointima was more heterogenous in PES than in SES. Miyoshi et al. [15] also reported more heterogeneous neointimal formation in PES compared to SES.

Moore et al. [20] compared polymer-coated rapamycineluting stents and non-polymer-coated rapamycin-eluting stents by OCT in patients, and they found that neointimal thickness was less and inhomogeneous in the former. This finding may suggest that polymers influence neointimal coverage. Further studies are required on whether regional differences in neointima thickness are caused by inhomogeneous coating of polymer, regional differences in concentration or release of drugs, or regional differences in vascular response.

\section{Significance of In-Stent Yellow Plaques}

Lesions without yellow color at baseline exhibited yellow color in $94 \%$ of SES-implanted lesions at follow-up of
10 months [3•]. Higo et al. [21] reported that the yellow color was due to formation of atherosclerotic neointima and that "yellow plaque" formation at the stented lesions was promoted by SES. However, since the in-stent "yellow plaques" were not examined histologically, the following other mechanisms may underlie this phenomenon: inflammatory cells (macrophages); polymer or polymer degrades infiltrated into the intima; deposition of $\beta$-carotene in these foreign substances; or organized thrombus.

\section{Peri-Strut Low-Density Area}

Miyoshi et al. [15] found by OCT that a peri-strut low-density area was more dominant in PES than in SES. Such a change has not been observed by angioscopy. Teramoto et al. [22] reported that peri-strut low-density area in a porcine model was due to fibrinoid and proteoglycans. It is necessary to examine whether the same substances or other mechanisms such as swollen polymers participate in this phenomenon.

\section{Late Stent Thrombosis}

Figure 2 shows an example of occlusive late stent thrombosis (LST) observed in a BMS. A similar occlusive LST can be formed in DES, resulting in acute coronary syndrome.

\section{BMS Versus DES (Table 2)}

LST was not observed in any patients 6 months after BMS implantation $[23,24]$. The prevalence of LST was com-

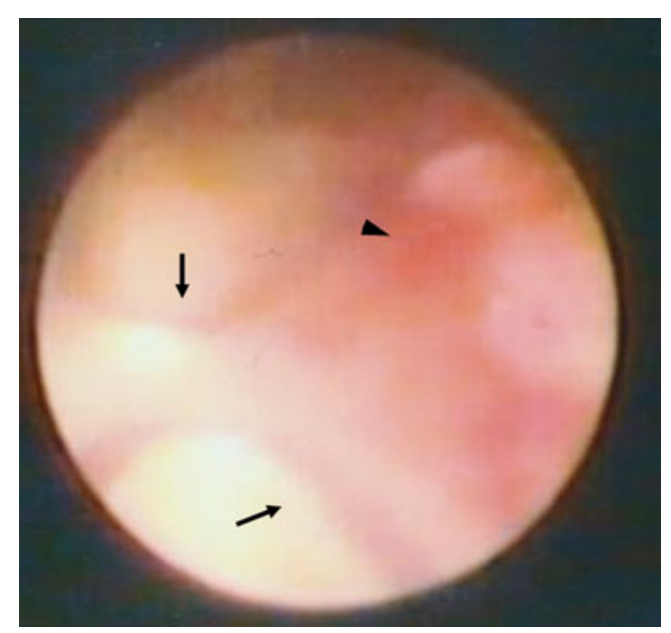

Fig. 2 A late stent thrombosis in the left anterior descending artery 6 months after deployment of a bare-metal stent in a 59-year-old man with ST-elevation myocardial infarction. Arrows indicate stent struts. The struts were seen through (grade 2 according to grading [5]). Arrowhead indicates thrombus 
Table 2 Incidence of LST by angioscopy

\begin{tabular}{lllll}
\hline & \multicolumn{4}{l}{ Incidence of LST, \% } \\
\cline { 2 - 5 } & BMS & SES & PES & ZES \\
\hline Fujimori et al. [23] & 0 & - & - & - \\
Tsukahara et al. [24] & 0 & - & - & - \\
Oyabu et al. [4] & 8 & 42 & - & - \\
Takano et al. [25] & 29 & 86 & - & - \\
Awata et al. [12] & - & 43 & 19 & - \\
Awata et al. [10••] & - & 31 & - & 6 \\
\hline
\end{tabular}

$B M S$ bare-metal stent, LST late stent thrombosis, PES paclitaxeleluting stent, $S E S$ sirolimus-eluting stent, ZES zotarolimus-eluting stent

pared between BMS and SES. The prevalence was significantly higher in the SES than in BMS (42-86\% vs $8-29 \%$ ) at 6 months after stent implantation [4, 25]. Thus, LST was less frequent in BMS, probably due to the higher prevalence of neointimal coverage.

\section{Among DES (Table 2)}

Awata et al. [12] observed that LST was more frequently observed in SES than in PES (43\% vs 19\%) at 6 months. They also reported that LST was observed in $31 \%$ of patients in the SES group versus $6 \%$ of patients in the ZES group at 6 months $[10 \bullet \bullet]$.

\section{Sensitivity of Angioscopy and OCT in Detecting LST}

Thrombus was observed in 12 of 24 SES and 8 of 28 PES by OCT, versus in 4 of SES and 4 of PES by angioscopy [11], indicating that OCT is more sensitive than angioscopy in detecting LST. One possible reason for this is that the entire stented lesion can be observed by OCT, while angioscopic observation is limited to a confined portion.

\section{Color of In-Stent Structure and LST}

Higo et al. [3•] observed that thrombus was more frequent on the yellow lesions than on the white lesions of the stented lesions in the chronic stage. They suggested that newly formed yellow intima is a potential risk of LST.

Takayama et al. [11] also observed that all LST was associated with yellow plaque within the stent, and a higher yellow grade was observed in SES. Thus, they suggested the characteristics of neointima tissue are different between SES and PES.

Whether or not yellow matter in the stented lesion is actually atheromatous plaque, or degraded polymer, or another substance remains unclear.

\section{Possible Mechanisms of LST and Appropriate Neointimal Thickening to Prevent Restenosis, Neo-endothelial Cell Damages, and Consequent LST}

Endothelial cells are highly anti-thrombotic. The same may also be true for neo-endothelial cells regenerated after stent implantation. Neointima is mainly composed of newly formed collagen fibers and located between neo-endothelial cells and stent struts, and acts as a cushion between neoendothelial cells and the stent struts.

If the neointima is thin, stent struts are considered to be dislocated synchronizing to blood pressure changes and cardiac motion, and accordingly induce mechanical stress on the neo-endothelial cells.

The struts were seen-through (grade $0-1$ ) by angioscopy when the neointima thickness was below $130 \mu \mathrm{m}$ by OCT in patients [6]. The LST was observed in grade 0 or 1 group in patients with DES or BMS $[10 \bullet \bullet, 26]$. At 6 months after stenting, neo-endothelial cells were stained in blue with Evans blue dye, which selectively stains damaged endothelial cells when the neointima thickness grade is 0 to 1 (Fig. 3) [26, 27]. In animals, the struts were visible (grade 0 or 1) when the neointimal thickness was around $88 \mu \mathrm{m}$, and LST was frequently observed when the intimal thickness was within $100 \mu \mathrm{m}$ [26]. All these findings indicated that neo-endothelial cells were damaged and LST formed on the struts when neointimal thickness was within approximately $100 \mu \mathrm{m}$ (Fig. 4).

Fresh LST has been observed in both patients and animals at 6 months after BMS deployment [26]. It is conceivable that even if the neo-endothelial cells were regenerated repeatedly, they were damaged repeatedly by friction between them and the struts due to luminal blood pressure changes and cardiac motion, and thus fresh thrombi were formed repeatedly even at 6 months or later. Determining exactly how to terminate this "vicious cycle" is an essential requisite for effective prophylactic treatment of LST.

LST was also observed when neointimal coverage was grade 0 or 1 in cases of SES and ZES [10••], as was the case for BMS [26]. A similar mechanism may underlie LST in the DES era.

Based on the results in these clinical and animal studies, the control of neointimal regeneration over $100 \mu \mathrm{m}$ and below an appropriate thickness that does not cause significant restenosis is an essential requisite for the prevention of LST and restenosis.

\section{New Stents and the Future}

Clinically available DES contain rapamycin, sirolimus, paclitaxel, or zotarolimus. Stent-based release of a selective 
Fig. 3 A stented segment of the left anterior descending artery 6 months after deployment of a bare-metal stent in a 69-year-old man with ST-elevation myocardial infarction. a A stent strut seen through (neointimal coverage: grade 2) before (arrow). b The same stent strut after Evans blue (arrow). The strut and the neighboring portions were stained blue, indicating damages of not only the neo-endothelial cells covering the struts but also those in neighboring portions. c Stent struts (neointimal coverage grade 2) distal to a (arrows). d The same struts (arrows). The stent struts and the neighboring portions were stained blue as in b, indicating extensive neoendothelial cell damage
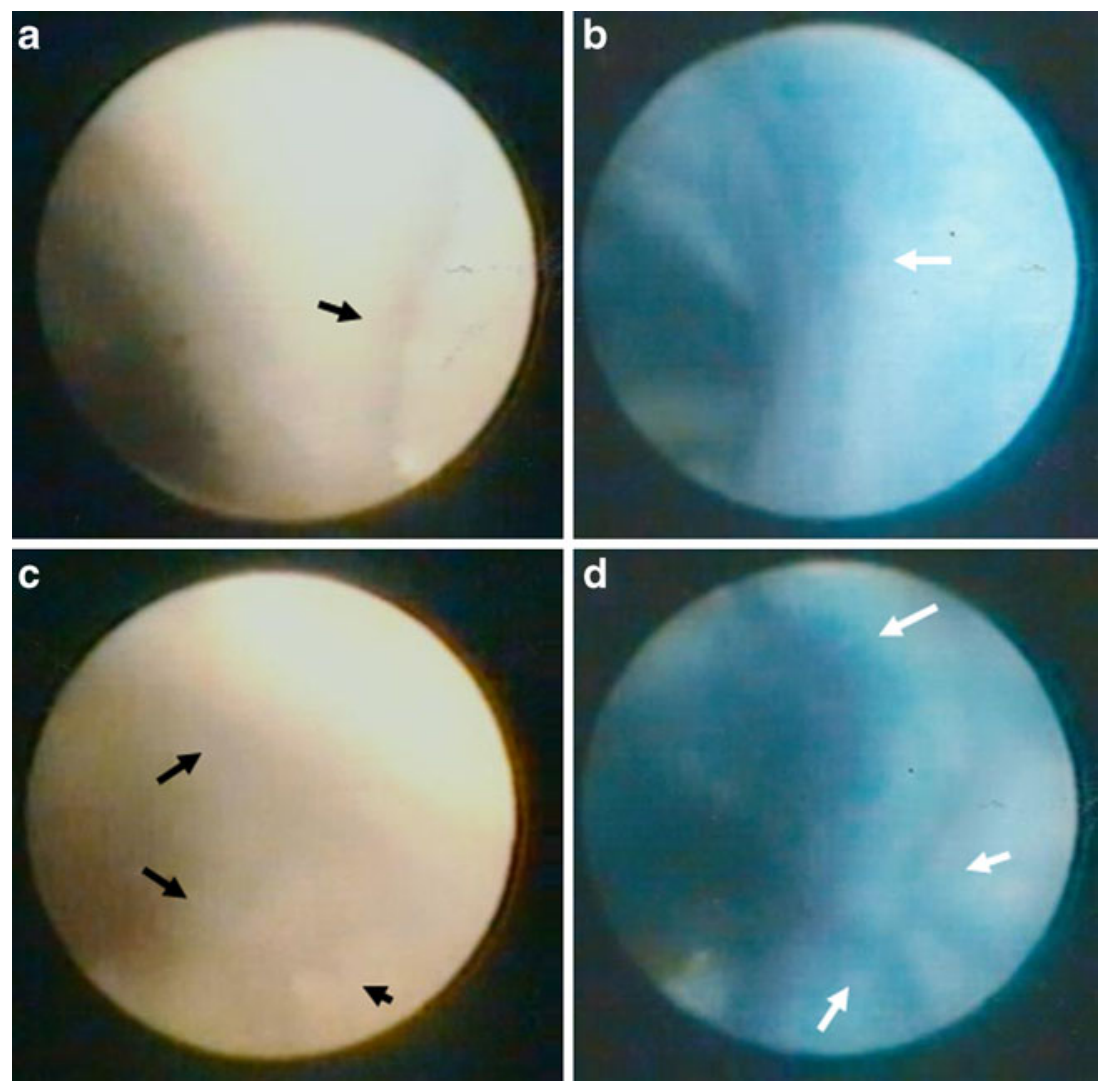

PDGF-receptor blocker from the bis-indolylmethanon class has been found to inhibit restenosis in a rabbit model [28]. Polyzene-F-coated stent, which prevents thrombosis, was also developed [29].

Recently, a DES that releases Evans blue dye slowly was developed because this dye prevents restenosis even after a single intracoronary administration [30].

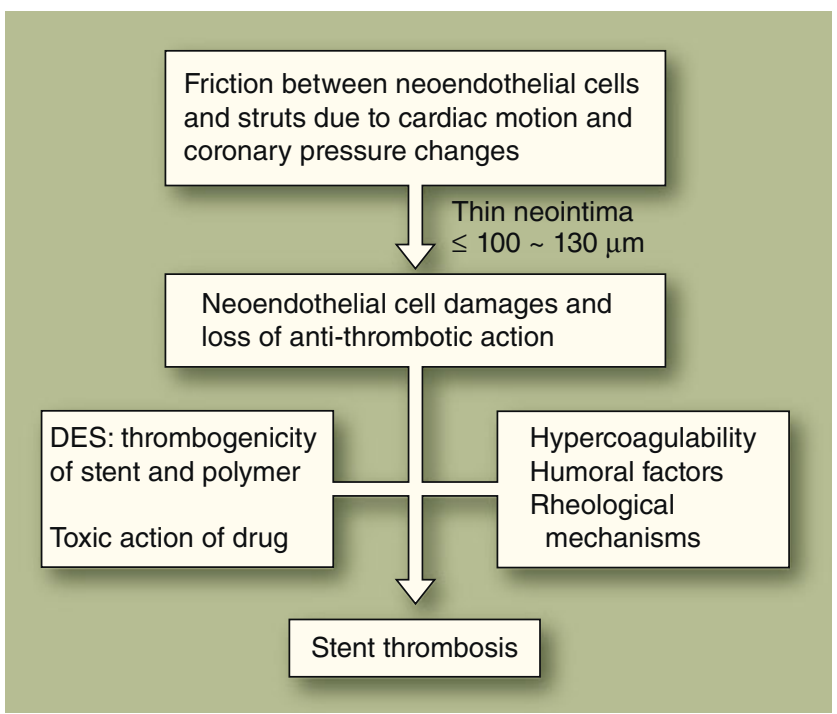

Fig. 4 Possible mechanisms for damage of the neo-endothelial cells covering stent struts. DES-drug-eluting stent
A drug-containing polymer is generally coated on the stent struts in DES. The polymer and its degraded products may influence intimal regeneration and neo-endothelial cell function. A polymer-free leflunomide-eluting stent was developed, and its efficacy was demonstrated in an animal model [31•].

Development of a new anti-thrombotic stent having the same elasticity as that of the neointima is another option for preventing LST. The major problems still to be resolved are as follows: 1) control of the neointima thickness; 2) acceleration of neo-endothelial cell coverage; and 3) prevention of neo-endothelial cell damage.

\section{Conclusions}

DES reduce coronary restenosis significantly; however, LST occurs, which requires long-term antiplatelet therapy. Angioscopic grading of neointimal coverage of coronary stent struts was established, and it was revealed that neointimal formation is incomplete and prevalence of LST is higher in DES when compared to BMS. It was also observed that the neointima is thicker and LST is less frequent in PES and ZES than in SES.

Endothelial cells are highly anti-thrombotic. Clinical and animal studies have revealed that neo-endothelial cells on stent struts are damaged when the neointima is less than 
$100 \mu \mathrm{m}$ thick, and LST frequently occurs. Neo-endothelial cell damage is considered to be caused by friction between the cells and stent struts due to the thin neointima between them, which might act as a cushion. Therefore, it is conceivable that controlling neointimal regeneration over $100 \mu \mathrm{m}$ and below appropriate thickness that does not cause significant restenosis is necessary to prevent LST. Also, development of a DES that has the same degree of elasticity as that of the neointima is a potential option with which to prevent neo-endothelial cell damage and consequent LST.

Disclosure No potential conflicts of interest relevant to this article were reported.

Open Access This article is distributed under the terms of the Creative Commons Attribution Noncommercial License which permits any noncommercial use, distribution, and reproduction in any medium, provided the original author(s) and source are credited.

\section{References}

Papers of particular interest, published recently, have been highlighted as:

- Of importance

•- Of major importance

1. Inoue T, Node K: Molecular basis of restenosis and novel issues of drug-eluting stents. Circ J 2009, 73:615-621.

2. Uchida Y: Coronary Angioscopy. Armonk, NY: Futura Publishing Company; 2001:83-91.

3. - Higo T, Ueda Y, Oyabu J, et al.: Atherosclerotic and thrombogenic neointima formed over SES. JACC Cardiovasc Imaging 2009, 2:616-624. The authors reported that yellow portions in the stented lesion are more thrombogenic than white portions in chronic phase.

4. Oyabu J, Ueda Y, Ogasawara N, et al.: Angioscopic evaluation of neointimal coverage: sirolimus drug-eluting stent versus bare metal stent. Am Heart J 2006, 52:1168-1174.

5. Kotani J, Awata M, Nanto S, et al.: Incomplete neointimal coverage of sirolimus-eluting stents: angioscopic findings. J Am Coll Cardiol 2006, 47:2108-2111.

6. Tsujimoto $\mathrm{T}$ : Relationships between angioscopic images of coronary stents and neointimal thickness measured by OCT. Proceedings of the 20th Annual Meeting of the Japanese Cardioangioscopy Society. 2006:30.

7. Sakai S, Mizuno K, Yokoyama S, et al.: Morphologic changes in infarct-related plaque after coronary stent placement. J Am Coll Cardiol 2003, 42:1558-1565.

8. Oyabu J, Ueda Y, Ogasawara N, et al.: Angioscopic evaluation of neointimal coverage: sirolimus drug-eluting stent versus bare metal stent. Am Heart J 2006, 52:1168-1174

9. Awata M, Kotani J, Uematsu M, et al.: Serial angioscopic evidence of incomplete neointimal coverage after SES implantation. Circulation 2007, 116:910-916.
10. •• Awata M, Nanto S, Uematsu M, et al.: Angioscopic comparison of neointimal coverage between zotarolimus- and sirolimuseluting stents. J Am Coll Cardiol 2008, 52:789-790. The authors demonstrated differences in neointimal coverage of stent struts and LST between ZES and SES.

11. Takayama T, Hiro $T$, Akabane $M$, et al.: Heterogenecity of coverage and color of neointima after drug-eluting stent implantation: observation by angioscopy and OCT. Circulation 2009, 120:S968.

12. Awata M, Nanto S, Uematsu M, et al.: Heterogenous arterial healing in patients following paclitaxel-eluting stent implantation. J Am Coll Cardiol Intv 2009, 2:453-458.

13. Ishii $\mathrm{N}$, Takayama $\mathrm{T}$, Honye $\mathrm{J}$, et al.: Investigation of neointimal coverage grading and yellow plaque grading in stent site after SES implantation-angioscopic finding. Circ J 2008, 72(Suppl 1):546.

14. Suzuki Y, Ikeno F, Koizumi T, et al.: In vivo comparison between optical coherence tomography and intravascular ultrasound for detecting small degree of in-stent neointima after stent implantation. JACC Cardiovasc Intv 2008, 1:168 173.

15. Miyoshi N, Shite J, Shnke T, et al.: Comparison by optical coherence tomography of paclitaxel-eluting stents with sirolimuseluting stents implanted in one coronary artery in one procedure. Circ J 2010, In press.

16. Sera $F$, Awata $M$, Uematsu $M$, et al.: Optimal stent-sizing with intravascular ultrasound contributes to complete neointimal coverage after SES implantation assessed by angioscopy. J Am Coll Cardiol Intv 2009, 2:989-994.

17. Chieffo A, Foglieni C, Nodari RL, et al.: Histopathology of clinical coronary restenosis in drug-eluting versus bare metal stents. Am J Cardiol 2009, 104:1660-1667.

18. Wilson GJ, Nakazawa G, Schwartz RS, et al.: Comparison of inflammatory response after implantation of sirolimus- and paclitaxel-eluting stents in porcine coronary arteries. Circulation 2009, 120:141-149.

19. Kim JS, Kim JS, Kim TH, et al.: Comparison of neointimal coverage of sirolimus-eluting stents and paclitaxel-eluting stents using optical coherence tomography at 9 months after implantation. Circ J 2010, 74:320-326.

20. Moore P, Barlis P, Spiro J, et al.: A randomized optical coherence tomography study of coronary stent strut coverage and luminal protrusion with rapamycin-eluting stents. JACC Cardiovasc Interv 2009, 2:437-444.

21. Higo T, Hirotani N, Ogasawara K, et al.: Sirolimus-eluting stent promotes formation of atherosclerotic neointima and yellow plaque at stented lesion: serial angioscopic study. Presented at the 81st Meeting of the American Heart Association. New Orleans, LA; November 2008.

22. Teramoto $\mathrm{T}$, Ikeno $\mathrm{F}$, Otake $\mathrm{H}$, et al.: Intriguing peri-strut lowdensity area detected by optical coherence tomography after coronary stent deployment. Circ J 2010, In press.

23. Fujimori Y, Morio H, Terasawa K, et al.: Angioscopic follow-up study on stented plaques in patients with acute myocardial infarction. Proceedings of the 13th Annual Meeting of Laser and Cardioangioscopy Society. 1999:28.

24. Tsukahara R, Hou M, Muramatsu T: Characteristics of coronary plaques culprit for acute myocardial infarction in chronic stage [in Japanese]. Coronary 1998, 15:145-150.

25. Takano $\mathrm{M}$, Ohba $\mathrm{T}$, Inami $\mathrm{S}$, et al.: Angioscopic differences in neointimal coverage and in persistence of thrombus between sirolimus-eluting stents and bare-metal stents after 6-month implantation. Eur Heart J 2006, 27:2189-2195.

26. Uchida Y, Uchida Y, Fujimori Y: Endothelial cells covering coronary stents are frequently damaged. Circulation 2006, 114 (Suppl II):591. 
27. Uchida Y, Nakamura F, Tomaru T: Observation of atherosclerotic lesions by an intravascular microscope in patients with arteriosclerosis obliterance. Am Heart J 1995, 130:11141117.

28. Jandt E, Mutschke O, Mahboobi S, et al.: Stent-based release of a selective PDGF-receptor blocker from the bis-indolylmethanon class inhibits restenosis in the rabbit animal model. Vascul Pharmacol 2010, 52:55-62.

29. La Manna A, Capodanno D, Cara M, et al.: Optical coherence tomographic results at six-month follow-up evaluation of the
CATANINA coronary stent system with nanothin polyzene-F surface modification (from the Assessment of the Latest NonThrombogenic Angioplasty Stent [ATLANTA] trial). Am J Cardiol 2009, 103:1551-1555.

30. Uchida $\mathrm{Y}$, Uchida $\mathrm{H}$, inventors: Therapeutic tool for vascular disease. US patent US7025981 B2. April 10, 2006.

31. - Deuse T, Erben RG, Ikeno F, et al.: Introducing the first polymer-free leflunomide eluting stent. Atherosclerosis 2008, 200:126-134. The authors developed a polymer-free stent. 